\title{
The Impact of International and Domestic Goods Trade Fluctuations on Shanghai's Economy: A Regional CGE Model-based Scenario Analysis
}

\author{
Lin Sun \\ Shanghai Academy of Social Sciences \\ 215, 7/622, Huaihai Zhong Lu, Huangpu District \\ Shanghai, China
}

\begin{abstract}
This paper examines the effect of international trade and domestic goods trade on the Shanghai economy using a dynamic computable general equilibrium (DCGE) model. The model distinguishes fourteen industry sectors, new dataset based on the 2015 input-output table, and offers simulations for the 2019-2030 periods. The simulation results indicate that the growth of goods export to the domestic market will promote the growth of real GDP of Shanghai economy, but the growth of goods export to the international market has the opposite effect. Simultaneously, the goods trade with the domestic market has more influence on Shanghai economy than international market. That is mean Shanghai economy has a closer relationship with the domestic market than the international market, has the ability to cope with fluctuations in the international market.
\end{abstract}

Keywords: Shanghai Economy, International Trade, Domestic Trade, CGE Model

\section{Introduction}

From March 2018, China and the United States are engaged in a trade war as each country continues to dispute tariffs placed on goods traded between them. After months of hostilities, a breakthrough of sorts came in December 2018. Both countries agreed to delay their planned increases in tariffs for 90 days, starting on December 1, to allow time for the two countries to negotiate their trade disputes. But after 20 March 2019, the negotiation is still ongoing. Trade wars have a major impact on the Chinese Economy, there has been some research studying related issues. Huang (2018) analyzed the Influence and Trend of the Upgrading of Sino-US Trade War, Wang (2018) analyzed the impact of the deteriorated Sino-US economic and trade relations on the Chinese Economy, Zhong (2018) analyzed the long-term impact of the trade war on the Chinese economy, Zhou et al (2018) analyzed the Impact on China's Economy and China's Countermeasures, Zhu\& Cao (2019) studied the overall impact of the decrease of US import demand and the deterioration of terms of trade on China's trade balance, financial stability, and real economy by a dynamic stochastic general equilibrium model.

Due to differences in economic development levels and economic structures across China, it is possible to assume that trade wars have different effects on different regions. As China's most important region, Shanghai economy is closely related to international trade (ROW) and other parts of the country (ROC), it is conceivable that the Shanghai economy will be more affected by the trade war than the inland areas. In recent years, there has been less literature of empirical research on the relationship with international trade and domestic trade, especially domestic trade. Li and Zhao (2005) used a VAR model to analyze the relation between ex-import and economic development of Shanghai and demonstrates the ways and importance of directly and indirectly promoting effects of import and export on economic growth. Sun \&Islam (2017) analyzed the economic relationships of Shanghai with the rest of China and the world by a CGE model Analysis. The purpose of this paper is to further analyze the effect of the change of goods trade on the Shanghai economy based on a new dataset. For this purpose, the paper uses a dynamic computable general equilibrium model (DCGE) that is based on the input-output data for 2015 and distinguishes fourteen industry sectors, capturing both demand and supply side linkages. Simulations are conducted for 2019 to 2030 . The paper is organized as follows. Section 2 describes the Dynamic CGE model that is constructed and used for the analysis. Section 3 presents the results. Section 4 concludes.

\section{A Dynamic Regional CGE Model for the Shanghai Economy}

CGE models designed to study development issues received considerable impetus from the work by Dervis, de Melo, and Robinson (1982). These models were later extended to study regional economies within or in relation with other economies. Thus, Madden (1990) developed a dynamic regional CGE model of Australia as two regions of an economy. Over time, regional CGE models have become a popular tool to study regional economies and related policy issues. Sun (2005), Sun and Islam (2017) also developed a single region model of Shanghai to study the effect of the Yuan 
exchange rate and the linkage with ROW and ROC. The dynamic regional computable general equilibrium (CGE) model used in this paper is a further application focusing on the effects of goods trade activity on Shanghai economy with a new dataset based on 2015 input-output table and different industry distinctions

\subsection{The basic Structure and Feature of Model for the Shanghai economy}

Different from national CGE models, regional CGE models have a more complex structure allowing for cross-region flows of products, factors, and funds. These cross-region flows can be endogenous, exogenous, or both (in part), depending on the model construction, which in turn depends on the purpose of the model. The Shanghai regional CGE model presented in this paper distinguishes fourteen industries, one type household (including rural and urban household), one type labor (including skilled and unskilled labor), and two types of government (central and regional). The basic structure and features of the model are presented in Sun and Islam (2017).

On the production side, all industries are assumed to operate under constant returns to the scale and observe the cost minimization rule. Production processes are assumed to follow CES (Constant Elasticity of Substitution) functions, the CES specification is used to combine labor and capital to produce the value added. The intermediate input requirement is determined through the use of Leontief type fixed coefficients applied to the gross output. On the supply side, the constant elasticity of transformation (CET) function is used to allow for substitution possibilities at two levels. At the initial level, the CET specification is used to allow substitution between exports and the domestically disposed of part of the output. The latter is disaggregated at the next level using the CET specification between the part that is marketed within Shanghai and the part that is marketed in ROC. On the demand side, CES specifications are used to conduct a similar two-level disaggregation with substitution possibilities.

On the demand side, at the first level, the CES specification is used to aggregate the demand for Shanghai-produced output and the demand for ROC-produced output into a combined demand for domestically produced output. At the second level, the CES specification is used again to aggregate the demand for domestic output and the demand for import, following the Armington assumption. So far as prices of exports and imports are concerned, Shanghai is assumed to a small-economy both ROC and ROW, making these prices exogenous to the Shanghai economy. Utility functions of the Cobb-Douglas type are used to model the consumption demand of residents and of regional and central governments. On the other hand, investment demand of the private sector and regional and central governments and the demand for intermediate output are all determined by fixed coefficients determined on the basis of the input-output table. The income of the private sector is determined by factor income, fewer taxes imposed on factor income (in the form of personal and property taxes). The income of the central government consists of indirect taxes, tariffs, personal income tax, enterprise income tax, less by transfers to the regional government. The income of the regional government consists of its share in the indirect taxes, personal income tax, enterprise income tax, and transfers from the central government. Households, the central government, and the local government each split up its income into consumption and savings. Savings of these three actors add up to form the total saving, which is spent on investment.

For overall equilibrium of the model, equilibrium has to be reached in seven different kinds of markets, namely (i) markets for Shanghai's own product inside Shanghai, (ii) markets for Shanghai's export to ROC, (iii) markets for Shanghai's export to ROW, (iv) markets in Shanghai for imports from ROC, (v) markets in Shanghai for imports from ROW, (vi) the market for labor, and finally (vii) the market for capital. Note that so far as product markets are concerned (i.e. the first five of the above list), fourteen separate markets need to be distinguished, each for the fourteen different industries. Equilibrium in each of the markets can be attained through adjustment of prices and/or quantities, depending on the assumptions. In the domestic product markets for Shanghai's own output, prices and output are jointly (endogenously) determined. However, given that prices in the markets for exports to and imports from both ROC and ROW are taken to be exogenous (following the small economy assumption), equilibrium in these markets is attained through adjustment of quantities only. The total supply of capital is given by the accumulation (investment) in each year added to previous year's capital stock and less by the depreciation. The rental rate adjusts to establish equality between the supply and the total demand for capital arising from the industries. Given the assumption of the mobility of capital across industries, each industry employs capital up to the point where the marginal value product of capital of the industry equals the equilibrium rental rate.

A well-specified model should satisfy Walras Law, according to which in an $n$-variable system, the equilibrium in $n-1$ markets should ensure the equilibrium in the $n$-th market. There are several popular ways to check whether Walras Law is satisfied in a CGE model. The model in this paper uses for this purpose the aggregate savings-investment equation, namely $S+F+F O-I^{n}=0$, in nominal terms, where $S$ denotes savings generated within Shanghai, and $F$ and $F O$ denote savings flowing in from ROC and ROW, respectively. $I^{n}$, on the other hand, denotes the total investment that Shanghai carries out either inside Shanghai or in ROC and ROW. Leaving out this equation also makes the price of savings as the numeraire. 
The model used in the paper is a dynamic recursive model so that it is solved for each year separately with updating equations connecting the model for one period with the one for the next period. The updating equations furnish the values (mostly through extrapolation) of the exogenous variables, such as the supply of labor, real investment by the private sector and by regional and central government, capital stock, and also the values of the parameters such as the total factor productivity of each industry, scale factors of exports and imports to or from ROC and ROW. Extrapolations are made on the basis of specific growth rates and changes assumed for the pertinent variables and parameters. The total capital stock is obtained by adding investment to the previous period's capital stock and subtracting the depreciation. The predetermined capital stock is then allocated among industries in accordance with the rate of return to capital prevailing in them.

\subsection{The Dataset and Baseline of Model for the Shanghai economy}

The baseline information is summarized in the form of the Social Accounting Matrix (SAM) presented in Table 1. The parameters of the model are calibrated on the basis of the information contained in this SAM. It shows the balance between demand and supply in the output market, the balance between aggregate savings and investment, the budgetary balance of various actors, and the balance in the transactions with ROC and ROW. The SAM presented in Table 1 is based on Shanghai's input-output table of 2015 and other macroeconomic and sectoral information pertaining to that year obtained from Shanghai Statistical Yearbook 2016. The model uses five kinds of elasticity pertaining to CES and CET functions. GTAP data is used to obtain the elasticity of substitution between labor and capital and between imports and domestic goods. The evidence available in other studies is used to obtain the elasticity of transformation between domestically disposed of output and export, and between export to ROC and ROW.

The first task in using the CGE model is to establish the baseline scenario (for 2015-2030) against which the simulated scenarios can be compared. The baseline needs to be reasonable, reflecting what would have happened if the recent trends by and large continued, and parameter values did not change too much. To construct such a baseline, it is assumed that during 2015-2030 labor, real investment, and TFP of each industry, respectively. These values accord well with the recent experience. Scale parameter of exports in each industry is extrapolated based on the growth performance of exports in the past. The exchange rate is fixed at the 2015 level, and the coefficients of intermediate inputs are assumed to remain the same as in the 2015 input-output table.

Table 1: Shanghai Social Accounting Matrix (SAM), 2015 in 100 Million Yuan

\begin{tabular}{|c|c|c|c|c|c|c|c|c|c|c|c|}
\hline Expenditures & 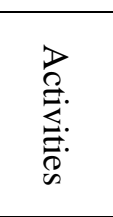 & $\begin{array}{l}\text { Q } \\
0 \\
0 \\
0 \\
0 \\
0 \\
0 \\
0\end{array}$ & 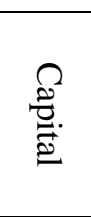 & एँ⿱艹 & 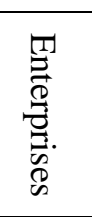 & $\begin{array}{l}\frac{T}{O} \\
0 \\
0 \\
0 \\
0 \\
0 \\
0 \\
0\end{array}$ & 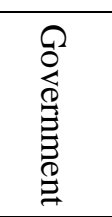 & 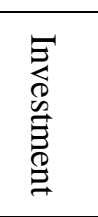 & 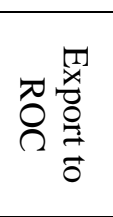 & 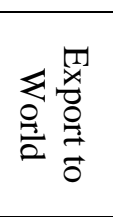 & $\stackrel{\overrightarrow{0}}{\stackrel{0}{0}}$ \\
\hline Activities & & 30588 & & & & & & & 33460 & 15384 & 79432 \\
\hline Commodities & 53772 & & & & & 11226 & 3765 & 9553 & & & 78315 \\
\hline Capital & 9699 & & & & & & & & & & 9699 \\
\hline Labor & 10836 & & & & & & & & & & 10836 \\
\hline Enterprises & & & 9699 & & & & & & & & 9699 \\
\hline Households & & & & 7233 & 6842 & & & & & & 14075 \\
\hline Government & 5125 & & & 3603 & 2857 & & & & & & 11585 \\
\hline Savings & & & & & & 2849 & 7820 & & -4410 & 3292 & 9553 \\
\hline Import from & & 29050 & & & & & & & & & 29050 \\
\hline Import from & & 18677 & & & & & & & & & 18677 \\
\hline Total & 79432 & 78315 & 9699 & 10836 & 9699 & 14075 & 11585 & 9553 & 29050 & 18677 & \\
\hline
\end{tabular}

Table 2 presents the baseline scenario in terms of average growth rates of key macroeconomic variables and gross value added by industry during 2015-2030. As we can see, under the baseline scenario, Shanghai's real GDP is to grow at an annual average rate of 6.4 percent. Shanghai's real import from ROW is to grow at a rate of 8.7 percent per annum, while real import from ROC is to grow at a rate of 8.5 percent per annum. On the other hand, Shanghai's export to ROC is to grow at an annual average rate of 8.2 percent, the rate at which Shanghai's export to ROW is 8.1 . The baseline scenario, therefore, reflects the current trend of the increasing dependence of Shanghai on ROW as a source of consumption and ROC as a source of demand for her output. 
Table 2: Baseline of Shanghai Economy, 2015-2020 (10 Million Yuan) ${ }^{1}$

\begin{tabular}{|c|c|c|c|c|c|c|c|c|c|c|c|c|c|c|c|c|}
\hline & 15 & 016 & 2017 & 018 & 019 & 2020 & 021 & 2022 & 023 & 2024 & 025 & 2026 & 2027 & 2020 & 2029 & 2030 \\
\hline NG & 256 & 786 & 029 & 296 & 591 & 917 & 276 & 672 & 109 & 592 & 124 & 712 & 361 & 077 & 868 & 740 \\
\hline DP & 6 & & 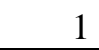 & & 0 & 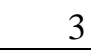 & 2 & & 5 & 0 & & & & & & \\
\hline $\mathrm{P}$ & .00 & 1.02 & 1.04 & 1.06 & 1.08 & 1.11 & 1.13 & 1.16 & 1.19 & 1.23 & 1.26 & 1.30 & 1.35 & 1.39 & 1.44 & 1.49 \\
\hline $\mathrm{RG}$ & 56 & 39 & 23 & 119 & 325 & 544 & 776 & 021 & 280 & 553 & 841 & 145 & 466 & 803 & 157 & 529 \\
\hline DP & 60 & & 9 & 1 & 8 & 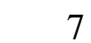 & 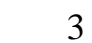 & 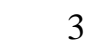 & 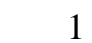 & . & 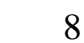 & 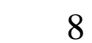 & & & 4 & \\
\hline \multirow[b]{2}{*}{$\mathrm{C}$} & 112 & 09 & 305 & 410 & 525 & 651 & 790 & 942 & 108 & 291 & 492 & 711 & 952 & 217 & 506 & 824 \\
\hline & $\angle 0$ & & & 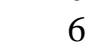 & 7 & 0 & 4 & 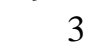 & 0 & 6 & 0 & 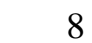 & 0 & & 0 & 7 \\
\hline GR & $\begin{array}{r}376 \\
5\end{array}$ & 957 & 162 & 383 & 621 & 878 & 154 & 452 & 774 & 6122 & 497 & 5903 & 342 & 816 & 330 & 885 \\
\hline \multirow{2}{*}{ IR } & 55 & 03 & 53 & 05 & 61 & 19 & 280 & 44 & 411 & 81 & 56 & 633 & 715 & 801 & 891 & 98. \\
\hline & 3 & & & & 1 & 2 & 1 & 1 & 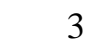 & 9 & 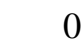 & & 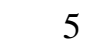 & & 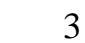 & 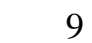 \\
\hline \multirow{2}{*}{ EOR } & 334 & 28 & 33 & 262 & 618 & 001 & 415 & 862 & 344 & 864 & 425 & 030 & 682 & 385 & 014 & 1096 \\
\hline & 6 & & 4 & 7 & 1 & 7 & 7 & 4 & 4 & 3 & 1 & 0 & $T$ & S & 45 & 25 \\
\hline \multirow{2}{*}{ ER } & 153 & 567 & 806 & 956 & 118 & 292 & 481 & 584 & 904 & 140 & 395 & 670 & $\overline{966}$ & 286 & 630 & 5002 \\
\hline & 84 & & & 3 & 2 & 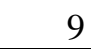 & 4 & 7 & 0 & $\tau$ & 5 & & & 4 & & 2 \\
\hline \multirow{2}{*}{$\begin{array}{c}\mathrm{MO} \\
\mathrm{R}\end{array}$} & 290 & $\overline{44}$ & 04 & 88 & 96 & 33 & 599 & $\longdiv { 9 7 }$ & 531 & 04 & 519 & 80 & 691 & 356 & 081 & 871 \\
\hline & 50 & 1 & 6 & 1 & 9 & 0 & 1 & 0 & $y$ & T & 6 & 5 & 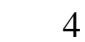 & & & 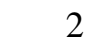 \\
\hline \multirow{2}{*}{ MR } & & 20 & 86 & 366 & 562 & 75 & 007 & 59 & 534 & 332 & 156 & 510 & 894 & 312 & 767 & 263 \\
\hline & 7 & 2 & 1 & 4 & 5 & 7 & 6 & 7 & 0 & 4 & 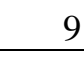 & 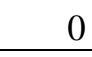 & 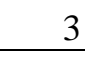 & & & 0 \\
\hline I1 & 304 & 328 & 354 & 381 & 411 & 443 & 477 & 514 & 554 & 597 & 643 & 693 & 747 & 80 & 860 & 933 \\
\hline $\mathrm{I} 2$ & 7 & 8 & 8 & 9 & 10 & 10 & 11 & 12 & 13 & 1 & 15 & 16 & 1 & 19 & 2 & 21 \\
\hline & & 40 & 47 & 79 & 39 & 28 & 250 & 06 & 199 & 732 & 309 & 332 & 606 & 334 & 012 & $\overline{097}$ \\
\hline & 74 & & & & 4 & 9 & 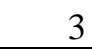 & & 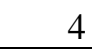 & 7 & 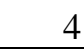 & 6 & & & 06 & 02 \\
\hline I4 & $\begin{array}{r}160 \\
5\end{array}$ & 730 & 65 & 11 & 168 & 38 & 0 & 17 & 9 & 58 & 4 & 70 & 6 & 54 & 6 & 4784 \\
\hline \multirow{2}{*}{ I5 } & 603 & & 91 & & 124 & & 243 & 88 & & & $\longdiv { 1 8 }$ & 074 & 133 & 195 & 261 & 331 \\
\hline & 4 & & 191 & 140 & & & & 88 & 158 & 050 & 3 & 0 & 0 & & & \\
\hline \multirow{2}{*}{ I6 } & 757 & (0) & 11 & & 24 & 03 & 89 & 80 & 79 & 85 & 599 & 722 & 354 & 995 & 147 & 2310 \\
\hline & 4 & & & & & & & 0 & 1 & 8 & 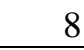 & J & 0 & 4 & 5 & 9 \\
\hline \multirow[b]{2}{*}{ I7 } & 556 & & & & 7537 & & 8767 & & 019 & 098 & 84 & 276 & 376 & 483 & 598 & 1722 \\
\hline & 0 & & & & 7 & & $8 / 67$ & & & 8 & & 8 & 2 & 2 & 5 & 6 \\
\hline I8 & $\begin{array}{r}121 \\
3\end{array}$ & 311 & 1417 & 531 & 1654 & 786 & 1929 & 083 & 2248 & 2427 & 2619 & 2826 & 3050 & 3290 & 3549 & 3828 \\
\hline I9 & $\begin{array}{r}316 \\
9\end{array}$ & 2 & 10 & 52 & 4110 & 35 & 678 & 91 & 325 & 5680 & 060 & 465 & 6898 & 7360 & 7854 & $x$ \\
\hline \multirow{2}{*}{ I10 } & 641 & 88 & 7 & 42 & 29 & & 83. & 56 & 34 & 17 & 307 & 404 & 508 & 520 & 739 & 368 \\
\hline & & & & & & & 903 & & & & & 6 & & 0 & & \\
\hline I1 1 & $\begin{array}{r}261 \\
2\end{array}$ & 778 & 55 & 2 & 3341 & 53 & 3778 & 4018 & 4273 & 4544 & 833 & 5141 & 5470 & 5819 & 6192 & 6590 \\
\hline \multirow{2}{*}{ I1 2} & 664 & & & & & & 005 & 76 & 153 & 235 & 23 & 418 & 519 & 627 & 743 & 868 \\
\hline & & & & & & & & & 5 & 7 & 7 & 1 & 3 & 7 & 9 & 5 \\
\hline 11 & 70 & & & & & & 098 & 183 & 276 & 376 & 1483 & 600 & 1726 & 862 & 2009 & 2168 \\
\hline \multirow{2}{*}{ I14 } & 601 & & 770 & & & & 63 & & & 1043 & 1112 & 1186 & 1266 & 1351 & 1444 & 1543 \\
\hline & & & 170 & & & & & 9193 & & & & 4 & & $y$ & 2 & \\
\hline
\end{tabular}

${ }^{1}$ NGDP: Nominal GDP; PGDP: GDP deflator; RGDP: Real GDP; CR: Real house consumption; GR: Real government consumption; IR: Real Investment; EOR: Rael Export to ROC; ER: Real Export to ROW; MOR: Rael Import from ROC; MR: Real Import from ROW;

I1: Agriculture; I2: Mining; I3: Manufacture; I4: Water, electricity and gas industry; I5: Construction; I6: Wholesale and retail trade; I7: Transportation and logistics; I8: hospitality industry; I9: Information software service; I10: Finance and insurance; I11: Real estate industry; I12: Lease and business service; I13: Residential service; I14: Public service. 


\section{The Simulations and their results}

\subsection{Simulations}

In order to analyze the effects of international goods trade (with ROW) and domestic trade (with ROC) on the Shanghai economy, simulations are designed to focus on the changes in trade in goods. First, approaching from the side of international trade, simulations are conducted to examine the influence on the Shanghai economy of changes in manufacture's exports and imports to or from ROW. Secondly, from the side of domestic trade, simulations are conducted to examine the influence on the Shanghai economy of changes in manufacture's exports and imports to or from ROC. Eight simulations are conducted, and these allow one percent increase or decrease in manufacture's export and import to or from ROW and one percent increase or decrease in manufacture's export and import to or from ROC, from 2019 to 2030 .

\subsection{Simulation Results}

Table 3 to table 10 presents the highlights of the simulation results in a normalized form in order to make the comparison easier. Look at the results of the simulations of the effects from the changes of international trade with a decrease or increase of export or import from ROW (table 3 to table 6).

It may be noticed that the $1 \%$ decrease in Shanghai manufacture's export to ROW (see table 3 ) has a positive effect on her real GDP and a significant negative impact on nominal GDP and GDP deflator, and the degree of effect is gradually expanding. Simultaneously, the decrease of goods export to ROW leads to a significant decrease of the whole Shanghai's import from ROW and ROC, and a weak increase of export to ROC, and the output of other industries are increasing.

Table 3: 1\% Decrease in Shanghai Manufacture's Export to ROW (compare with baseline, \%)

\begin{tabular}{|c|c|c|c|c|c|c|c|c|c|c|c|c|}
\hline & 2019 & 2020 & 2021 & 2022 & 2023 & 2024 & 2025 & 2026 & 2027 & 2028 & 2029 & 2030 \\
\hline NGDP & -0.30 & -0.92 & -1.85 & -3.07 & -4.56 & -6.28 & -8.18 & 10.22 & 12.35 & 14.52 & 16.69 & 18.81 \\
\hline PGDP & -0.39 & -1.17 & -2.35 & -3.90 & -5.77 & -7.91 & 10.26 & 12.74 & 15.28 & $17.82^{-}$ & $20.30^{-}$ & 22.66 \\
\hline RGDP & 0.08 & 0.25 & 0.51 & 0.86 & 1.29 & 1.78 & 2.32 & 2.88 & 3.45 & 4.01 & 4.52 & 4.97 \\
\hline CR & -0.19 & -0.59 & -1.18 & -1.97 & -2.94 & -4.06 & -5.31 & -6.67 & -8.09 & -9.55 & 11.02 & 12.47 \\
\hline GR & 0.04 & 0.11 & 0.20 & 0.30 & 0.40 & 0.50 & 0.58 & 0.64 & 0.67 & 0.68 & 0.66 & 0.60 \\
\hline IR & -0.17 & -0.52 & -1.05 & -1.74 & -2.6 & -3.6 & -4.71 & -5.93 & -7.22 & -8.56 & -9.92 & -11.3 \\
\hline EOR & 0.00 & 0.02 & 0.04 & 0.09 & 0.14 & 0.22 & 0.31 & 0.41 & 0.52 & 0.64 & 0.76 & 0.89 \\
\hline ER & -0.23 & -0.70 & -1.39 & -2.31 & -3.45 & -4.79 & -6.34 & -8.08 & -9.99 & 12.08 & 14.31 & 16.68 \\
\hline MOR & .14 & .42 & 0.84 & -1.39 & -2.06 & 2.82 & .68 & -4.61 & -5.58 & -6.59 & -7.60 & -8.61 \\
\hline MR & -0.18 & 4 & 07 & -1.76 & -2.60 & .57 & 4.65 & -5.82 & -7.05 & -8.32 & -9.61 & 10.89 \\
\hline I1 & 0.12 & 0.36 & 0.73 & 1.22 & 1.83 & 2.55 & 3.37 & 4.29 & 5.28 & 6.33 & 7.43 & 8.57 \\
\hline $\mathrm{I} 2$ & 0.14 & 0.44 & 0.89 & 1.49 & 2.24 & 3.14 & 4.16 & 5.30 & 6.54 & 7.86 & 9.24 & 10.66 \\
\hline I3 & -0.16 & -0.47 & -0.92 & -1.50 & -2.19 & -2.98 & -3.85 & -4.80 & -5.80 & -6.83 & -7.87 & -8.90 \\
\hline I4 & 0.01 & 0.04 & 0.09 & 0.16 & 0.24 & 0.34 & 0.46 & 0.59 & 0.73 & 0.88 & 1.03 & 1.19 \\
\hline I5 & 0.00 & 0.01 & 0.01 & 0.02 & 0.04 & 0.05 & 0.07 & 0.09 & 0.12 & 0.15 & 0.18 & 0.22 \\
\hline I6 & 0.02 & 0.06 & 0.13 & 0.23 & 0.36 & 0.54 & 0.77 & 1.03 & 1.34 & 1.68 & 2.06 & 2.46 \\
\hline I7 & 0.11 & 0.35 & 0.70 & 1.16 & 1.74 & 2.41 & 3.17 & 4.01 & 4.91 & 5.85 & 6.83 & 7.81 \\
\hline I8 & 0.12 & 0.38 & 0.76 & 1.27 & 1.91 & 2.66 & 3.52 & 4.46 & 5.49 & 6.57 & 7.70 & 8.85 \\
\hline I9 & 0.12 & 0.36 & 0.72 & 1.19 & 1.78 & 2.48 & 3.27 & 4.13 & 5.06 & 6.04 & 7.04 & 8.06 \\
\hline I10 & 0.05 & 0.14 & 0.28 & 0.47 & 0.70 & 0.98 & 1.30 & 1.65 & 2.03 & 2.43 & 2.84 & 3.27 \\
\hline I11 & 0.09 & 0.27 & 0.54 & 0.89 & 1.33 & 1.83 & 2.39 & 3.01 & 3.66 & 4.34 & 5.03 & 5.71 \\
\hline $\mathrm{I} 12$ & 0.08 & 0.23 & 0.47 & 0.78 & 1.17 & 1.63 & 2.15 & 2.73 & 3.34 & 3.99 & 4.66 & 5.34 \\
\hline I13 & 0.04 & 0.11 & 0.23 & 0.37 & 0.55 & 0.75 & 0.97 & 1.21 & 1.46 & 1.72 & 1.98 & 2.24 \\
\hline I14 & 0.12 & 0.35 & 0.69 & 1.14 & 1.68 & 2.30 & 2.99 & 3.73 & 4.52 & 5.33 & 6.15 & 6.97 \\
\hline
\end{tabular}


In contrast, the $1 \%$ increase in Shanghai manufacture's export to ROW (see table 4) has a gradually expanding negative effect on her real GDP and a significant positive impact on nominal GDP and GDP deflator, and the degree of effect is gradually expanding. Simultaneously, the increase of goods export to ROW leads to a significant increase of the whole Shanghai's import from ROW and ROC, and the same degree of increase of export to ROC, the output of all other industries has been negatively affected to varying degrees.

Table 4: $1 \%$ Increase in Shanghai Manufacture's Export to ROW (compare with baseline, \%)

\begin{tabular}{|c|c|c|c|c|c|c|c|c|c|c|c|c|}
\hline & 2019 & 2020 & 2021 & 2022 & 2023 & 2024 & 2025 & 2026 & 2027 & 2028 & 2029 & 2030 \\
\hline NGDP & 0.31 & 0.94 & 1.94 & 3.34 & 5.21 & 7.60 & 10.61 & 14.34 & 18.91 & 24.49 & 31.25 & 39.42 \\
\hline PGDP & 0.39 & 1.21 & 2.51 & 4.39 & 6.95 & 10.35 & 14.79 & 20.57 & 28.10 & 37.98 & 51.13 & 69.01 \\
\hline RGDP & -0.08 & -0.26 & -0.56 & -1.01 & -1.63 & -2.49 & -3.64 & -5.17 & -7.17 & -9.78 & -13.16 & -17.51 \\
\hline $\mathrm{CR}$ & 0.19 & 0.60 & 1.23 & 2.13 & 3.31 & 4.84 & 6.74 & 9.10 & 11.96 & 15.43 & 19.61 & 24.63 \\
\hline GR & -0.04 & -0.11 & -0.19 & -0.29 & -0.37 & -0.43 & -0.45 & -0.40 & -0.25 & 0.04 & 0.49 & 1.17 \\
\hline IR & 0.17 & 0.53 & 1.08 & 1.86 & 2.89 & 4.20 & 5.84 & 7.86 & 10.32 & 13.30 & 16.88 & 21.17 \\
\hline EOR & 0.00 & -0.02 & -0.06 & -0.12 & -0.22 & -0.37 & -0.59 & -0.88 & -1.27 & -1.76 & -2.39 & -3.17 \\
\hline ER & 0.23 & 0.69 & 1.38 & 2.29 & 3.43 & 4.78 & 6.36 & 8.15 & 10.14 & 12.34 & 14.72 & 17.27 \\
\hline MOR & 0.14 & 0.43 & 0.87 & 1.46 & 2.24 & 3.20 & 4.39 & 5.81 & 7.52 & 9.55 & 11.96 & 14.81 \\
\hline MR & 0.18 & 0.54 & 1.09 & 1.84 & 2.81 & 4.01 & 5.48 & 7.24 & 9.35 & 11.86 & 14.83 & 18.36 \\
\hline I1 & -0.12 & -0.36 & -0.75 & -1.28 & -1.99 & -2.89 & -4.00 & -5.35 & -6.96 & -8.87 & -11.08 & -13.62 \\
\hline $\mathrm{I} 2$ & -0.14 & -0.44 & -0.91 & -1.56 & -2.41 & -3.50 & -4.83 & -6.45 & -8.37 & -10.61 & -13.21 & -16.17 \\
\hline I3 & 0.16 & 0.47 & 0.92 & 1.51 & 2.24 & 3.09 & 4.07 & 5.17 & 6.39 & 7.72 & 9.15 & 10.67 \\
\hline I4 & -0.01 & -0.05 & -0.10 & -0.18 & -0.30 & -0.47 & -0.68 & -0.96 & -1.32 & -1.76 & -2.31 & -2.95 \\
\hline I5 & 0.00 & -0.01 & -0.01 & -0.02 & $\begin{array}{l}-0.04 \\
\end{array}$ & -0.05 & -0.07 & -0.10 & -0.13 & -0.17 & -0.21 & -0.27 \\
\hline I6 & -0.02 & -0.06 & -0.12 & -0.21 & -0.33 & -0.47 & -0.64 & -0.84 & -1.04 & -1.25 & -1.47 & -1.67 \\
\hline I7 & -0.11 & -0.35 & -0.71 & -1.22 & -1.88 & -2.71 & -3.73 & -4.96 & -6.42 & -8.13 & -10.11 & -12.38 \\
\hline I8 & -0.12 & -0.38 & -0.78 & -1.32 & -2.03 & -2.92 & -4.00 & -5.28 & -6.80 & -8.55 & -10.55 & -12.82 \\
\hline I9 & -0.12 & -0.36 & -0.73 & -1.24 & -1.89 & -2.70 & -3.68 & -4.83 & -6.17 & -7.69 & -9.42 & -11.34 \\
\hline $\mathrm{I} 10$ & -0.05 & -0.14 & -0.28 & -0.48 & -0.73 & -1.04 & -1.40 & -1.82 & -2.30 & -2.84 & -3.44 & -4.09 \\
\hline I11 & -0.09 & -0.27 & -0.55 & -0.92 & -1.39 & -1.96 & -2.64 & -3.43 & -4.32 & -5.31 & -6.41 & -7.61 \\
\hline $\mathrm{I} 12$ & -0.08 & -0.23 & -0.48 & -0.82 & -1.26 & -1.82 & -2.50 & -3.32 & -4.29 & -5.41 & -6.72 & -8.22 \\
\hline I13 & -0.04 & -0.12 & -0.24 & -0.40 & -0.61 & -0.88 & -1.22 & -1.62 & -2.11 & -2.68 & -3.37 & -4.16 \\
\hline $\mathrm{I} 14$ & -0.12 & -0.35 & -0.70 & -1.18 & -1.78 & -2.50 & -3.37 & -4.37 & -5.53 & -6.84 & -8.31 & -9.94 \\
\hline
\end{tabular}

The 1\% decrease in Shanghai manufacture's import from ROW (see table 5) has a negative effect on her real GDP, nominal GDP and GDP deflator, and the degree of effect is gradually expanding. Simultaneously, the decrease of goods import from ROW leads to a slight decrease of the whole Shanghai's export to ROW and ROC, and an increase of import from ROC, and a slight effect on the output of other industries.

Table 5: 1\% Decrease in Shanghai Manufacture's Import from ROW (compare with baseline, \%)

\begin{tabular}{|c|c|c|c|c|c|c|c|c|c|c|c|c|}
\hline & 2019 & 2020 & 2021 & 2022 & 2023 & 2024 & 2025 & 2026 & 2027 & 2028 & 2029 & 2030 \\
\hline NGDP & -0.08 & -0.23 & -0.46 & -0.77 & -1.16 & -1.63 & -2.17 & -2.80 & -3.51 & \begin{tabular}{|l|}
-4.29 \\
\end{tabular} & -5.15 & -6.08 \\
\hline PGDP & -0.06 & -0.17 & -0.35 & -0.58 & -0.86 & -1.21 & -1.60 & -2.04 & -2.53 & -3.06 & -3.62 & -4.22 \\
\hline RGDP & $\begin{array}{l}-0.02 \\
\end{array}$ & -0.05 & -0.11 & -0.19 & -0.30 & -0.43 & $\begin{array}{c}-0.59 \\
\end{array}$ & $\begin{array}{l}-0.78 \\
\end{array}$ & -1.00 & -1.27 & -1.58 & -1.94 \\
\hline CR & -0.06 & -0.17 & $\begin{array}{l}-0.34 \\
\end{array}$ & -0.57 & -0.85 & -1.19 & $\begin{array}{l}-1.59 \\
\end{array}$ & -2.05 & -2.55 & \begin{tabular}{|l|}
-3.11 \\
\end{tabular} & -3.73 & -4.39 \\
\hline GR & 0.00 & 0.01 & 0.02 & 0.03 & 0.04 & 0.05 & 0.06 & 0.06 & 0.07 & 0.06 & 0.05 & 0.04 \\
\hline IR & 0.00 & 0.01 & 0.01 & 0.02 & 0.03 & 0.03 & 0.04 & 0.05 & 0.06 & 0.07 & 0.08 & 0.10 \\
\hline EOR & 0.00 & $\begin{array}{l}-0.01 \\
\end{array}$ & $\begin{array}{c}-0.02 \\
\end{array}$ & -0.03 & -0.05 & -0.06 & $\begin{array}{l}-0.08 \\
\end{array}$ & $\begin{array}{l}-0.10 \\
\end{array}$ & -0.12 & $\begin{array}{l}-0.14 \\
\end{array}$ & -0.16 & -0.18 \\
\hline ER & -0.01 & $\begin{array}{l}-0.02 \\
\end{array}$ & -0.05 & -0.08 & -0.11 & -0.16 & -0.20 & -0.25 & -0.31 & -0.37 & -0.43 & -0.50 \\
\hline MOR & 0.03 & 0.09 & 0.18 & 0.30 & 0.46 & 0.64 & 0.87 & 1.12 & 1.41 & 1.74 & 2.10 & 2.51 \\
\hline MR & -0.07 & $\begin{array}{l}-0.21 \\
\end{array}$ & -0.42 & -0.69 & -1.04 & -1.45 & -1.93 & $\begin{array}{l}-2.48 \\
\end{array}$ & -3.09 & -3.77 & -4.51 & -5.31 \\
\hline I1 & \begin{tabular}{l|l|}
-0.01 \\
\end{tabular} & $\begin{array}{l}-0.04 \\
\end{array}$ & -0.07 & -0.12 & $\begin{array}{l}-0.17 \\
\end{array}$ & -0.24 & $\begin{array}{l}-0.31 \\
\end{array}$ & $\begin{array}{l}-0.38 \\
\end{array}$ & -0.46 & -0.55 & -0.64 & -0.73 \\
\hline I2 & $\begin{array}{l}-0.02 \\
\end{array}$ & -0.07 & $\begin{array}{l}-0.13 \\
\end{array}$ & -0.21 & $\begin{array}{l}-0.32 \\
\end{array}$ & -0.44 & -0.57 & $\begin{array}{l}-0.73 \\
\end{array}$ & -0.90 & $\begin{array}{l}-1.08 \\
\end{array}$ & -1.29 & -1.50 \\
\hline I3 & \begin{tabular}{l|l|}
-0.01 \\
\end{tabular} & $\begin{array}{l}-0.04 \\
\end{array}$ & -0.07 & -0.11 & -0.17 & -0.23 & -0.29 & -0.37 & -0.44 & $\begin{array}{l}-0.53 \\
\end{array}$ & -0.61 & -0.70 \\
\hline I4 & 0.00 & 0.00 & 0.00 & -0.01 & $\begin{array}{l}-0.01 \\
\end{array}$ & -0.01 & 0.00 & 0.00 & 0.01 & 0.01 & 0.02 & 0.04 \\
\hline I5 & 0.00 & -0.01 & -0.02 & -0.04 & -0.06 & -0.09 & -0.12 & -0.17 & -0.21 & -0.27 & -0.33 & -0.41 \\
\hline I6 & 0.01 & 0.02 & 0.03 & 0.05 & 0.08 & 0.12 & 0.16 & 0.21 & 0.26 & 0.32 & 0.38 & 0.45 \\
\hline I7 & 0.01 & 0.02 & 0.04 & 0.07 & 0.10 & 0.14 & 0.19 & 0.25 & 0.31 & 0.38 & 0.46 & 0.55 \\
\hline I8 & 0.00 & $\begin{array}{l}-0.01 \\
\end{array}$ & $\begin{array}{c}-0.02 \\
\end{array}$ & -0.03 & $\begin{array}{l}-0.05 \\
\end{array}$ & -0.06 & -0.07 & $\begin{array}{l}-0.09 \\
\end{array}$ & -0.11 & $\begin{array}{l}-0.12 \\
\end{array}$ & -0.14 & -0.15 \\
\hline I9 & 0.01 & 0.03 & 0.07 & 0.11 & 0.16 & 0.23 & 0.30 & 0.38 & 0.48 & 0.58 & 0.69 & 0.80 \\
\hline I10 & 0.01 & 0.02 & 0.03 & 0.06 & 0.09 & 0.12 & 0.16 & 0.20 & 0.25 & 0.31 & 0.37 & 0.43 \\
\hline I11 & 0.01 & 0.04 & 0.07 & 0.12 & 0.18 & 0.24 & 0.32 & 0.40 & 0.49 & 0.59 & 0.69 & 0.80 \\
\hline I12 & 0.00 & 0.01 & 0.02 & 0.04 & 0.05 & 0.08 & 0.10 & 0.13 & 0.16 & 0.20 & 0.24 & 0.28 \\
\hline I13 & $\begin{array}{l}-0.01 \\
\end{array}$ & $\begin{array}{l}-0.02 \\
\end{array}$ & $\begin{array}{l}-0.03 \\
\end{array}$ & -0.05 & $\begin{array}{l}-0.08 \\
\end{array}$ & -0.11 & $\begin{array}{l}-0.15 \\
\end{array}$ & $\begin{array}{l}-0.19 \\
\end{array}$ & -0.23 & $\begin{array}{l}-0.28 \\
\end{array}$ & -0.34 & -0.40 \\
\hline I14 & 0.01 & 0.02 & 0.04 & 0.06 & 0.08 & 0.11 & 0.15 & 0.18 & 0.22 & 0.26 & 0.30 & 0.34 \\
\hline
\end{tabular}


In contrast, the $1 \%$ increase in Shanghai manufacture's import from ROW (see table 6) has a significant positive effect on her real GDP, nominal GDP and GDP deflator, and the degree of effect is gradually expanding. Simultaneously, the increase of goods import from ROW leads to a moderate decrease of the whole Shanghai's import from ROC, and the slight degree of increase of export to ROC and ROW has a slight effect on the output of all other industries.

Table 6: 1\% Increase in Shanghai Manufacture's Import from ROW (compare with baseline, \%)

\begin{tabular}{|c|c|c|c|c|c|c|c|c|c|c|c|c|}
\hline & 2019 & 2020 & 2021 & 2022 & 2023 & 2024 & 2025 & 2026 & 2027 & 2028 & 2029 & 2030 \\
\hline NGDP & 0.07 & 0.22 & 0.45 & 0.74 & 1.11 & 1.55 & 2.05 & 2.62 & 3.24 & 3.92 & 4.65 & 5.43 \\
\hline PGDP & 0.06 & 0.17 & 0.34 & 0.56 & 0.83 & 1.15 & 1.52 & 1.92 & 2.37 & 2.84 & 3.34 & 3.86 \\
\hline RGDP & 0.02 & 0.05 & 0.11 & 0.18 & 0.28 & 0.39 & 0.52 & 0.68 & 0.85 & 1.05 & 1.27 & 1.51 \\
\hline CR & 0.06 & 0.17 & 0.33 & 0.55 & 0.83 & 1.16 & 1.54 & 1.97 & 2.46 & 3.00 & 3.58 & 4.22 \\
\hline GR & 0.00 & $\begin{array}{l}-0.01 \\
\end{array}$ & -0.02 & -0.03 & -0.04 & -0.05 & -0.06 & -0.06 & -0.06 & -0.05 & -0.04 & -0.03 \\
\hline IR & 0.06 & 0.17 & 0.33 & 0.54 & 0.81 & 1.12 & 1.47 & 1.86 & 2.29 & 2.75 & 3.24 & 3.75 \\
\hline EOR & 0.00 & 0.01 & 0.02 & 0.03 & 0.05 & 0.06 & 0.08 & 0.09 & 0.11 & 0.12 & 0.14 & 0.15 \\
\hline ER & 0.01 & 0.02 & 0.05 & 0.08 & 0.11 & 0.15 & 0.19 & 0.23 & 0.28 & 0.33 & 0.38 & 0.42 \\
\hline MOR & -0.03 & -0.09 & -0.18 & -0.29 & -0.44 & -0.61 & -0.81 & -1.04 & -1.30 & -1.58 & -1.89 & -2.22 \\
\hline MR & 0.07 & 0.21 & 0.41 & 0.68 & 1.01 & 1.41 & 1.87 & 2.39 & 2.98 & 3.62 & 4.33 & 5.10 \\
\hline I1 & 0.01 & 0.04 & 0.07 & 0.11 & 0.16 & 0.22 & 0.28 & 0.34 & 0.40 & 0.46 & 0.52 & 0.57 \\
\hline $\mathrm{I} 2$ & 0.02 & 0.06 & 0.13 & 0.21 & 0.30 & 0.42 & 0.54 & 0.68 & 0.84 & 1.00 & 1.17 & 1.36 \\
\hline $\mathrm{I} 3$ & 0.01 & 0.04 & 0.07 & 0.11 & 0.16 & 0.21 & 0.27 & 0.34 & 0.40 & 0.47 & 0.54 & 0.61 \\
\hline I4 & 0.00 & 0.00 & 0.00 & 0.01 & 0.01 & 0.00 & 0.00 & 0.00 & -0.01 & -0.02 & -0.04 & -0.05 \\
\hline I5 & 0.00 & 0.01 & 0.02 & 0.04 & 0.06 & 0.09 & 0.12 & 0.16 & 0.20 & 0.25 & 0.31 & 0.38 \\
\hline I6 & $\begin{array}{l}-0.01 \\
\end{array}$ & $\begin{array}{l}-0.02 \\
\end{array}$ & $\begin{array}{l}-0.03 \\
\end{array}$ & -0.05 & -0.08 & -0.11 & -0.14 & $\begin{array}{l}-0.18 \\
\end{array}$ & -0.23 & -0.27 & -0.32 & $\begin{array}{l}-0.37 \\
\end{array}$ \\
\hline I7 & -0.01 & -0.02 & -0.04 & -0.06 & -0.10 & -0.14 & -0.18 & -0.23 & -0.29 & -0.36 & -0.43 & -0.50 \\
\hline I8 & 0.00 & 0.01 & 0.02 & 0.03 & 0.04 & 0.05 & 0.07 & 0.08 & 0.09 & 0.10 & 0.10 & 0.11 \\
\hline I9 & -0.01 & -0.03 & -0.06 & -0.11 & -0.16 & -0.21 & -0.28 & -0.36 & -0.44 & -0.52 & -0.62 & -0.71 \\
\hline $\mathrm{I} 10$ & -0.01 & -0.02 & -0.03 & -0.06 & -0.08 & -0.11 & -0.15 & -0.19 & -0.23 & -0.27 & -0.32 & -0.37 \\
\hline I11 & -0.01 & -0.04 & -0.07 & -0.11 & -0.17 & -0.23 & -0.30 & -0.37 & -0.45 & -0.53 & -0.61 & -0.70 \\
\hline $\mathrm{I} 12$ & 0.00 & -0.01 & -0.02 & -0.03 & -0.05 & -0.07 & -0.10 & -0.12 & -0.15 & -0.18 & -0.22 & -0.26 \\
\hline I13 & 0.01 & 0.02 & 0.03 & 0.05 & 0.08 & 0.10 & 0.14 & 0.17 & 0.21 & 0.24 & 0.28 & 0.33 \\
\hline I14 & -0.01 & -0.02 & -0.04 & -0.06 & -0.08 & -0.11 & -0.14 & -0.17 & -0.21 & -0.24 & -0.28 & -0.32 \\
\hline
\end{tabular}

Look at the results of the simulations of the effects from the changes of domestic trade with a decrease or increase of export or import from ROC (table 7 to table 10). It may be noticed that the $1 \%$ decrease in Shanghai manufacture's export to ROC (see table 7) has a positive effect on her real GDP and a significant negative impact on nominal GDP and GDP deflator, and the degree of effect is gradually expanding. Simultaneously, the decrease of goods export to ROC leads to a significant decrease of the whole Shanghai's import from ROW and ROC, and a moderate decrease of export to ROW, and a significant positive effect on the output of other industries.

Table 7: 1\% Decrease in Shanghai Manufacture's Export to ROC (compare with baseline, \%)

\begin{tabular}{|c|c|c|c|c|c|c|c|c|c|c|c|c|}
\hline & 2019 & 2020 & 2021 & 2022 & 2023 & 2024 & 2025 & 2026 & 2027 & 2028 & 2029 & 2030 \\
\hline NGDP & -0.49 & -1.47 & -2.94 & -4.86 & -7.19 & -9.86 & -12.80 & -15.93 & -19.15 & -22.38 & -25.56 & -28.62 \\
\hline PGDP & $\begin{array}{l}-0.62 \\
\end{array}$ & -1.86 & -3.73 & -6.14 & -9.04 & -12.34 & -15.91 & -19.63 & -23.40 & -27.09 & -30.63 & -33.95 \\
\hline RGDP & 0.13 & 0.40 & 0.82 & 1.37 & 2.03 & 2.82 & 3.69 & 4.61 & 5.55 & 6.46 & 7.32 & 8.07 \\
\hline CR & -0.31 & -0.93 & -1.88 & -3.12 & -4.64 & -6.41 & -8.37 & -10.47 & -12.66 & -14.89 & -17.11 & -19.27 \\
\hline GR & 0.06 & 0.17 & 0.32 & 0.49 & 0.66 & 0.84 & 1.01 & 1.16 & 1.29 & 1.39 & 1.45 & 1.47 \\
\hline IR & -0.27 & -0.83 & -1.66 & -2.76 & -4.11 & -5.70 & -7.47 & -9.40 & -11.43 & -13.53 & -15.65 & -17.75 \\
\hline EOR & -0.15 & -0.43 & -0.86 & -1.41 & -2.10 & -2.91 & -3.84 & -4.89 & -6.05 & -7.33 & -8.71 & -10.18 \\
\hline ER & -0.04 & -0.11 & -0.21 & -0.34 & -0.51 & -0.70 & -0.94 & -1.23 & -1.57 & -1.98 & -2.44 & -2.97 \\
\hline MOR & -0.23 & -0.68 & -1.34 & -2.22 & -3.29 & -4.53 & -5.92 & -7.43 & -9.03 & -10.69 & -12.39 & -14.09 \\
\hline MR & -0.29 & -0.86 & -1.70 & -2.81 & -4.16 & -5.73 & -7.50 & -9.42 & -11.46 & -13.59 & -15.77 & -17.96 \\
\hline I1 & 0.19 & 0.57 & 1.16 & 1.94 & 2.91 & 4.07 & 5.39 & 6.87 & 8.46 & 10.16 & 11.91 & 13.70 \\
\hline $\mathrm{I} 2$ & 0.23 & 0.70 & 1.41 & 2.38 & 3.58 & 5.03 & 6.71 & 8.58 & 10.63 & 12.82 & 15.13 & 17.50 \\
\hline $\mathrm{I} 3$ & -0.25 & -0.75 & $\begin{array}{l}-1.48 \\
\end{array}$ & -2.42 & -3.58 & -4.91 & -6.42 & -8.09 & -9.90 & -11.83 & -13.84 & -15.91 \\
\hline I4 & 0.02 & 0.07 & 0.14 & 0.24 & 0.36 & 0.51 & 0.67 & 0.83 & 1.00 & 1.16 & 1.31 & 1.44 \\
\hline I5 & 0.00 & 0.01 & 0.02 & 0.04 & 0.06 & 0.09 & 0.12 & 0.16 & 0.20 & 0.26 & 0.32 & 0.39 \\
\hline I6 & 0.03 & 0.09 & 0.21 & 0.39 & 0.62 & 0.94 & 1.35 & 1.86 & 2.48 & 3.21 & 4.05 & 5.00 \\
\hline I7 & 0.18 & 0.55 & 1.11 & 1.86 & 2.77 & 3.86 & 5.10 & 6.47 & 7.94 & 9.48 & 11.08 & 12.69 \\
\hline I8 & 0.20 & 0.60 & 1.22 & 2.04 & 3.07 & 4.29 & 5.71 & 7.29 & 9.01 & 10.85 & 12.79 & 14.78 \\
\hline I9 & 0.19 & 0.57 & 1.15 & 1.92 & 2.87 & 4.01 & 5.32 & 6.77 & 8.35 & 10.02 & 11.77 & 13.55 \\
\hline I10 & 0.07 & 0.22 & 0.45 & 0.76 & 1.14 & 1.61 & 2.14 & 2.75 & 3.42 & 4.14 & 4.89 & 5.68 \\
\hline I11 & 0.14 & 0.43 & 0.87 & 1.44 & 2.14 & 2.98 & 3.92 & 4.97 & 6.10 & 7.29 & 8.52 & 9.77 \\
\hline I12 & 0.12 & 0.37 & 0.75 & 1.26 & 1.88 & 2.63 & 3.48 & 4.42 & 5.44 & 6.53 & 7.66 & 8.82 \\
\hline I13 & 0.06 & 0.18 & 0.36 & 0.59 & 0.87 & 1.18 & 1.53 & 1.90 & 2.28 & 2.66 & 3.03 & 3.38 \\
\hline I14 & 0.19 & 0.56 & 1.11 & 1.83 & 2.70 & 3.72 & 4.86 & 6.11 & 7.44 & 8.83 & 10.25 & 11.68 \\
\hline
\end{tabular}


In contrast, the $1 \%$ increase in Shanghai manufacture's export to ROC (see table 8 ) has a significant negative effect on her real GDP and a significant positive impact on nominal GDP and GDP deflator, and the degree of effect is gradually expanding. Simultaneously, the increase of goods export to ROC leads to a significant increase of the whole Shanghai's import from ROW and ROC, and a moderate increase of export to ROW, and a significant negative impact on the output of all other industries.

Table 8: $1 \%$ Increase in Shanghai Manufacture's Export to ROC (compare with baseline, \%)

\begin{tabular}{|c|c|c|c|c|c|c|c|c|c|c|c|c|}
\hline & 2019 & 2020 & 2021 & 2022 & 2023 & 2024 & 2025 & 2026 & 2027 & 2028 & 2029 & 2030 \\
\hline NGDP & 0.49 & 1.50 & 3.10 & 5.36 & 8.37 & 12.24 & 17.13 & 23.19 & 30.62 & 39.68 & 50.62 & 63.79 \\
\hline PGDP & 0.62 & 1.93 & 4.03 & 7.07 & 11.25 & 16.85 & 24.29 & 34.12 & 47.23 & 64.96 & 89.56 & 125.05 \\
\hline RGDP & -0.13 & -0.42 & -0.89 & -1.60 & -2.59 & -3.95 & -5.76 & -8.15 & -11.28 & -15.33 & -20.54 & -27.22 \\
\hline CR & 0.31 & 0.95 & 1.97 & 3.40 & 5.31 & 7.75 & 10.82 & 14.59 & 19.18 & 24.71 & 31.34 & 39.24 \\
\hline GR & -0.06 & -0.17 & -0.30 & -0.44 & -0.56 & -0.62 & -0.59 & -0.42 & -0.07 & 0.53 & 1.44 & 2.73 \\
\hline IR & 0.27 & 0.84 & 1.73 & 2.97 & 4.61 & 6.70 & 9.31 & 12.51 & 16.39 & 21.06 & 26.65 & 33.31 \\
\hline EOR & 0.14 & 0.43 & 0.83 & 1.35 & 1.98 & 2.69 & 3.49 & 4.34 & 5.24 & 6.17 & 7.11 & 8.05 \\
\hline ER & 0.04 & 0.10 & 0.17 & 0.24 & 0.27 & 0.25 & 0.16 & -0.05 & -0.38 & -0.88 & -1.57 & -2.46 \\
\hline MOR & 0.22 & 0.68 & 1.37 & 2.32 & 3.54 & 5.06 & 6.92 & 9.14 & 11.79 & 14.93 & 18.65 & 23.04 \\
\hline MR & 0.29 & 0.86 & 1.73 & 2.92 & 4.44 & 6.32 & 8.61 & 11.35 & 14.61 & 18.49 & 23.08 & 28.53 \\
\hline I1 & -0.19 & -0.58 & -1.19 & -2.03 & -3.15 & -4.54 & -6.26 & -8.30 & -10.70 & -13.46 & -16.57 & -20.02 \\
\hline $\mathrm{I} 2$ & -0.23 & -0.70 & -1.44 & -2.46 & -3.81 & -5.49 & -7.53 & -9.96 & -12.79 & -16.02 & -19.63 & -23.62 \\
\hline I3 & 0.25 & 0.74 & 1.45 & 2.37 & 3.48 & 4.76 & 6.19 & 7.75 & 9.43 & 11.19 & 13.02 & 14.89 \\
\hline I4 & -0.02 & -0.07 & -0.16 & -0.30 & -0.49 & -0.77 & -1.12 & -1.58 & -2.15 & -2.85 & -3.67 & -4.63 \\
\hline I5 & 0.00 & -0.01 & -0.02 & -0.04 & -0.06 & -0.08 & -0.11 & -0.15 & -0.19 & -0.24 & -0.31 & -0.39 \\
\hline I6 & -0.03 & -0.09 & -0.18 & -0.32 & -0.49 & -0.69 & -0.92 & -1.15 & -1.38 & -1.60 & -1.80 & -1.97 \\
\hline I7 & -0.18 & -0.56 & -1.14 & -1.94 & -2.97 & -4.27 & -5.84 & -7.71 & -9.88 & -12.38 & -15.19 & -18.31 \\
\hline I8 & -0.20 & -0.60 & -1.23 & -2.09 & -3.19 & -4.56 & -6.20 & -8.11 & -10.31 & -12.79 & -15.55 & -18.56 \\
\hline I9 & -0.19 & -0.57 & -1.15 & -1.95 & -2.97 & -4.21 & -5.68 & -7.39 & -9.31 & -11.45 & -13.80 & -16.32 \\
\hline I10 & -0.07 & -0.22 & -0.45 & -0.75 & -1.14 & -1.60 & -2.14 & -2.74 & -3.42 & -4.14 & -4.92 & -5.74 \\
\hline I11 & -0.14 & -0.43 & -0.87 & -1.45 & -2.18 & -3.05 & -4.06 & -5.20 & -6.46 & -7.83 & -9.28 & -10.81 \\
\hline $\mathrm{I} 12$ & -0.12 & -0.37 & -0.76 & -1.30 & -2.00 & -2.86 & -3.91 & -5.15 & -6.58 & -8.23 & -10.09 & -12.17 \\
\hline I13 & -0.06 & -0.19 & -0.38 & -0.64 & -0.98 & -1.41 & -1.93 & -2.57 & -3.32 & -4.19 & -5.21 & -6.37 \\
\hline I14 & -0.19 & -0.56 & -1.12 & -1.86 & -2.79 & -3.90 & -5.20 & -6.69 & -8.36 & -10.20 & -12.21 & -14.38 \\
\hline
\end{tabular}

The 1\% decrease in Shanghai manufacture's import from ROC (see table 9) has a significant negative effect on her real GDP, nominal GDP and GDP deflator, and the degree of effect is gradually expanding. Simultaneously, the decrease of goods import from ROC leads to a slight decrease of the whole Shanghai's export to ROW and ROC, and a significant increase of import from ROW, and a moderate effect on the output of other industries.

Table 9: 1\% Decrease in Shanghai Manufacture's Import from ROC (compare with baseline, \%)

\begin{tabular}{|c|c|c|c|c|c|c|c|c|c|c|c|c|}
\hline & 2019 & 2020 & 2021 & 2022 & 2023 & 2024 & 2025 & 2026 & 2027 & 2028 & 2029 & 2030 \\
\hline NGDP & -0.50 & -1.52 & -3.05 & -5.09 & -7.64 & -10.67 & -14.17 & -18.10 & -22.43 & -27.11 & -32.09 & -37.30 \\
\hline PGDP & -0.24 & -0.72 & -1.43 & -2.40 & -3.60 & -5.06 & -6.76 & -8.71 & -10.91 & -13.35 & -16.04 & -18.95 \\
\hline RGDP & -0.27 & -0.81 & -1.64 & -2.76 & -4.19 & -5.91 & -7.95 & -10.29 & -12.94 & -15.88 & -19.12 & -22.64 \\
\hline $\mathrm{CR}$ & -0.23 & -0.69 & -1.39 & -2.30 & -3.43 & -4.76 & -6.28 & -7.95 & -9.77 & -11.71 & -13.74 & -15.84 \\
\hline GR & 0.02 & 0.05 & 0.09 & 0.13 & 0.18 & 0.22 & 0.25 & 0.28 & 0.29 & 0.29 & 0.27 & 0.23 \\
\hline IR & 0.01 & 0.03 & 0.05 & 0.08 & 0.11 & 0.15 & 0.19 & 0.24 & 0.30 & 0.36 & 0.43 & 0.51 \\
\hline EOR & -0.02 & -0.05 & -0.09 & -0.14 & -0.21 & -0.27 & -0.35 & -0.43 & -0.51 & -0.60 & -0.69 & -0.78 \\
\hline ER & $\begin{array}{l}-0.04 \\
\end{array}$ & -0.10 & -0.20 & -0.33 & -0.48 & -0.65 & -0.85 & -1.07 & -1.31 & -1.56 & -1.83 & -2.12 \\
\hline MOR & -0.08 & -0.23 & -0.46 & -0.77 & -1.16 & -1.64 & -2.22 & -2.91 & -3.70 & -4.61 & -5.65 & -6.82 \\
\hline MR & 0.27 & 0.81 & 1.63 & 2.73 & 4.10 & 5.76 & 7.71 & 9.93 & 12.45 & 15.24 & 18.32 & 21.67 \\
\hline I1 & -0.05 & -0.16 & -0.31 & -0.50 & -0.74 & -1.02 & -1.34 & -1.70 & -2.10 & -2.53 & -3.00 & -3.50 \\
\hline $\mathrm{I} 2$ & -0.09 & -0.27 & -0.53 & -0.88 & -1.30 & -1.79 & -2.34 & -2.96 & -3.62 & -4.34 & -5.10 & -5.90 \\
\hline $\mathrm{I} 3$ & -0.05 & -0.15 & -0.29 & -0.48 & -0.69 & -0.94 & -1.22 & -1.53 & -1.86 & -2.21 & -2.58 & -2.97 \\
\hline $\mathrm{I} 4$ & 0.00 & -0.01 & -0.02 & -0.03 & -0.03 & -0.04 & -0.04 & -0.04 & -0.03 & -0.03 & -0.01 & 0.00 \\
\hline I5 & $\begin{array}{l}-0.02 \\
\end{array}$ & -0.05 & -0.10 & -0.17 & -0.26 & -0.37 & -0.50 & -0.66 & -0.84 & -1.05 & -1.29 & -1.55 \\
\hline I6 & 0.02 & 0.07 & 0.14 & 0.23 & 0.36 & 0.51 & 0.69 & 0.91 & 1.16 & 1.44 & 1.76 & 2.11 \\
\hline I7 & 0.03 & 0.08 & 0.16 & 0.27 & 0.41 & 0.57 & 0.77 & 0.98 & 1.23 & 1.50 & 1.79 & 2.09 \\
\hline I8 & -0.02 & -0.04 & -0.09 & -0.14 & -0.20 & -0.27 & -0.34 & -0.43 & -0.52 & -0.62 & -0.73 & -0.84 \\
\hline I9 & 0.05 & 0.14 & 0.27 & 0.45 & 0.67 & 0.93 & 1.23 & 1.57 & 1.95 & 2.35 & 2.79 & 3.24 \\
\hline $\mathrm{I} 10$ & 0.02 & 0.07 & 0.15 & 0.24 & 0.36 & 0.51 & 0.67 & 0.86 & 1.08 & 1.31 & 1.56 & 1.84 \\
\hline I11 & 0.05 & 0.15 & 0.30 & 0.50 & 0.73 & 1.01 & 1.33 & 1.68 & 2.06 & 2.47 & 2.91 & 3.37 \\
\hline I12 & 0.01 & 0.04 & 0.09 & 0.15 & 0.22 & 0.31 & 0.41 & 0.53 & 0.66 & 0.81 & 0.96 & 1.12 \\
\hline I13 & -0.02 & -0.07 & -0.14 & -0.23 & -0.34 & -0.48 & -0.64 & -0.82 & -1.03 & -1.27 & -1.52 & -1.80 \\
\hline I14 & 0.03 & 0.08 & 0.15 & 0.24 & 0.34 & 0.46 & 0.58 & 0.71 & 0.85 & 0.98 & 1.11 & 1.24 \\
\hline
\end{tabular}


In contrast, the 1\% increase in Shanghai manufacture's import from ROC (see table 10) has a significant positive effect on her real GDP, nominal GDP and GDP deflator, and the degree of effect is gradually expanding. Simultaneously, the increase of goods import from ROC leads to a significant decrease of the whole Shanghai's import from ROW, and the slight degree of increase of export to ROC and ROW has a moderate effect on the output of all other industries.

Table 10: 1\% Increase in Shanghai Manufacture's Import from ROC (compare with baseline, \%)

\begin{tabular}{|c|c|c|c|c|c|c|c|c|c|c|c|c|}
\hline & 019 & 2020 & 2021 & 2022 & 2023 & 2024 & 2025 & 2026 & 2027 & 2028 & 2029 & 2030 \\
\hline NGDP & 0.50 & 1.51 & 3.02 & 5.06 & 7.61 & 10.68 & 14.27 & 18.37 & 22.97 & 28.05 & 33.58 & 39.53 \\
\hline PGDP & 23 & 70 & 37 & .23 & 3.25 & 4.39 & 5.60 & 6.82 & 8.00 & 9.05 & 9.91 & 10.50 \\
\hline RGDP & 26 & 80 & .63 & 2.76 & 4.22 & 6.03 & 8.21 & 10.81 & 13.87 & 7.42 & 1.53 & 26.26 \\
\hline CR & 0.23 & 0.69 & 1.39 & 2.34 & 3.54 & 5.01 & 6.75 & 8.80 & 11.16 & 13.87 & 16.96 & 20.46 \\
\hline GR & .02 & 0.05 & -0.09 & -0.13 & -0.17 & -0.20 & -0.22 & -0.23 & -0.22 & -0.18 & -0.12 & -0.04 \\
\hline IR & .01 & -0.02 & -0.04 & -0.07 & -0.09 & -0.12 & -0.14 & -0.16 & -0.18 & -0.20 & -0.21 & -0.22 \\
\hline EOR & 02 & 05 & .09 & 0.14 & 0.19 & 0.25 & 0.31 & 0.37 & 0.43 & 0.49 & 0.54 & 0.58 \\
\hline ER & & 10 & $(0$ & 31 & .45 & .61 & .77 & 0.95 & .13 & .32 & .51 & 1.70 \\
\hline MOR & 7 & 22 & & 70 & .02 & 1.39 & 1.79 & 2.21 & 2.64 & 3.07 & 3.47 & 3.85 \\
\hline MR & 27 & 80 & 50 & 60 & 92 & 3 & 17 & -9.12 & 11.25 & 13.56 & 16.01 & 18.58 \\
\hline I1 & 0.05 & .15 & 0.29 & 0.46 & 0.66 & 0.87 & 1.08 & 1.30 & 1.50 & 1.68 & 1.84 & 1.97 \\
\hline $\mathrm{I} 2$ & 0.09 & 0.27 & 0.53 & 0.86 & 1.27 & 1.75 & 2.30 & 2.91 & 3.57 & 4.30 & 5.07 & 5.89 \\
\hline I3 & 0.05 & 0.15 & 0.29 & 0.46 & 0.66 & 0.88 & 1.12 & 1.38 & 1.65 & 1.92 & 2.19 & 2.46 \\
\hline I4 & 00 & 01 & 02 & .02 & 0.02 & 0.00 & -0.02 & -0.05 & -0.09 & -0.15 & -0.23 & -0.32 \\
\hline I5 & 01 & 0.05 & .10 & 16 & 0.26 & 0.37 & 0.51 & 0.68 & 0.88 & 1.11 & 1.38 & 1.69 \\
\hline I6 & 02 & 06 & 13 & 21 & 32 & .44 & .58 & -0.72 & -0.88 & -1.04 & -1.20 & -1.36 \\
\hline I7 & -0.03 & -0.08 & -0.16 & -0.27 & -0.41 & -0.58 & -0.78 & -1.01 & -1.26 & -1.55 & -1.86 & -2.19 \\
\hline I8 & 0.02 & 0.04 & 0.08 & 0.12 & 0.17 & 0.21 & 0.25 & 0.28 & 0.30 & 0.30 & 0.30 & 0.28 \\
\hline I9 & -0.04 & -0.13 & -0.27 & -0.44 & -0.65 & -0.90 & -1.18 & -1.49 & -1.83 & -2.19 & -2.58 & -2.97 \\
\hline I10 & -0.02 & -0.07 & -0.14 & -0.23 & $\begin{array}{l}-0.34 \\
\end{array}$ & -0.47 & $\begin{array}{l}-0.61 \\
\end{array}$ & -0.76 & -0.93 & -1.10 & -1.28 & -1.46 \\
\hline I11 & .05 & -0.15 & -0.29 & -0.48 & -0.69 & -0.94 & -1.22 & -1.52 & -1.83 & -2.15 & -2.48 & -2.82 \\
\hline $\mathrm{I} 12$ & -0.01 & -0.04 & -0.09 & -0.14 & -0.22 & -0.31 & -0.41 & -0.52 & -0.65 & -0.79 & -0.94 & -1.09 \\
\hline $\mathrm{I} 13$ & & & & 0.21 & & 0.42 & 0.54 & 0.67 & 0.81 & 0.94 & 1.08 & 1.22 \\
\hline I14 & -0.03 & -0.08 & -0.15 & -0.24 & -0.35 & -0.47 & -0.60 & -0.75 & -0.91 & -1.08 & -1.26 & -1.44 \\
\hline
\end{tabular}

\section{Conclusions}

Results from simulations focusing on changes in the international and domestic goods trade with ROW and ROC show that the change of international goods trade and domestic goods trade has a different effect on the Shanghai economy. Firstly, about the international goods trade, from the perspective of the impact on GDP, the decrease in Shanghai manufacture's export to ROW has a positive effect on real GDP and a significant negative impact on nominal GDP; in contrast, the increase of the export to ROW has a negative effect on real GDP and a significant positive impact on nominal GDP; the decrease of the import from ROW has a negative effect on real and nominal GDP; in contrast, the increase of the import from ROW has a significant positive effect on real and nominal GDP. About the domestic goods trade, the decrease in Shanghai manufacture's export to ROC has a positive effect on real GDP and a significant negative impact on nominal GDP, in contrast, the increase of the export to ROC has a significant negative effect on real GDP and a significant positive impact on nominal GDP; the decrease of the import from ROC has a significant negative effect on real and nominal GDP, in contrast, the increase of the import from ROC has a significant positive effect on real and nominal GDP. Secondly, in terms of the extent of influence to Shanghai economy, the simulation results show that the change of domestic goods trade with ROW has a greater effect on Shanghai economy than the change of international goods with ROW based on the same extent change of manufacture's goods trade. Thirdly, the simulation results show that the change of manufacture's goods export has a greater effect on Shanghai economy than import in international trade, but has the similar extent effect of the export and import in domestic trade.

These results show that the growth of goods export to the domestic market will promote the growth of real GDP of Shanghai economy, but the growth of goods export to the international market has the opposite effect. Simultaneously, the goods trade with the domestic market has more influence on Shanghai economy than international market. That is mean Shanghai economy has a closer relationship with the domestic market than the international market and has the ability to cope with fluctuations in the international market. 


\section{References}

Dervis K., J. de Melo, and S. Robinson, The General Equilibrium Models for Development Policy, Cambridge: Cambridge University Press, 1982.

Huang Lijian, The Cause, Influence and Trend Analysis of the Upgrading of Sino-US Trade War, New Finance, No.9, 2018 (In Chinese).

LiHuizhongand Zhao Da-ping, The Empirical Analysis of the Relation between Ex-import and Economic Development of Shanghai, Fudan Journal (Social Sciences), No.6, 2005 (In Chinese).

Madden, J. R., FEDERAL: A Two-Region Multisectoral Fiscal Model of the Australian Economy, Ph.D. Thesis, Hobart: University of Tasmania, 1990.

Sun Lin, The Linkage of Shanghai Economy with the Rest of China and the World Market - Input-Output and CGE Model Analysis, 15th International Conference on Input-Output Techniques, Beijing, China, June 27 to July $1,2005$.

Sun Lin and Nazrul Islam, Economic Relationships of Shanghai with the Rest of China and the World: A CGE Model Analysis, International Journal of Business and Social Science, Volume 8, Number 6, 2017.

Wang Binrong, Impact of the Deteriorated Sino-US Economic and Trade Relations on the Chinese Economy, Frontiers, No. 16, 2018 (In Chinese).

ZhongZhengsheng, The long-term impact of the trade war on the Chinese economy, China Reform, No.6, 2018 (In Chinese).

Zhou Xiaobo, Song Liyi and Ni Kun, The US Trade War with China: The Impact on China's Economy and China's Countermeasures, New, Finance, No.6, 2018 (In Chinese).

ZhuMengnanand Cao Chunyu, Sino - US Trade War and Exchange R ate Arrangement Choice: A Policy Simulation on Dynamic Stochastic General Equilibrium Model, Finance and Trade Research, No.2, 2019 (In Chinese). 